\title{
Análisis espacio-temporal de la ocurrencia de incendios forestales en Durango, México
}

\section{Spatial-temporal analysis of fire occurrence in Durango, Mexico}

\author{
Gustavo Pérez-Verdín ${ }^{1 *}$, Marco Antonio Márquez-Linares ${ }^{1}$, \\ Armando Cortés-Ortiz ${ }^{1}$ y Maricela Salmerón-Macías ${ }^{1}$
}

\begin{abstract}
RESUMEN
Los incendios forestales representan un gran problema en la pérdida de biodiversidad, en la emisión de gases efecto invernadero y en la modificación de los flujos hídricos. En México, los incendios son causados en su mayoría por la acción del hombre, por lo que factores como la accesibilidad, distancia a caminos y poblados, entre otros, influyen en su frecuencia y distribución. Utilizando una base de datos de la Conafor, periodo 2000-2011, se realizó un análisis espacio-temporal de la ocurrencia de los incendios forestales en Durango, uno de los estados con mayor afectación en el país. Se utilizó el índice de Moran para determinar un patrón de distribución espacial y se hizo un análisis de estacionalidad y autocorrelación temporal utilizando los datos colectados. Para determinar los factores más importantes que inciden en el tamaño de los incendios, se aplicó la regresión geográficamente ponderada cuya característica principal es la asignación de niveles de importancia (peso geográfico) en función de la distancia. Los resultados indican que los incendios forestales se distribuyen de manera agregada, son no-estacionarios y no están correlacionados temporalmente. El tamaño de los incendios se manifiesta de acuerdo con la densidad de población y vías de acceso, lo que confirma la importancia del factor antropogénico en la magnitud de estos eventos. Bajas precipitaciones y altas temperaturas son factores climáticos que también afectan en gran medida la ocurrencia de los incendios. De manera general y considerando el factor antropogénico, se requieren más apoyos en educación y capacitación para reducir los efectos del fuego.
\end{abstract}

PALABRAS CLAVE:

Autocorrelación, estacionalidad, regresión por pesos geográficos, regímenes de propiedad, bosques de clima templado.

\begin{abstract}
Forest fires affect biodiversity, increase emission of greenhouse gasses, and modify hydrological flows. In Mexico, fires are mostly caused by humans and factors like road accessibility, distance to towns, among others are commonly associated with their frequency and distribution. Using a Conafor database collected from 2000 to 2011, a spatial-temporal evaluation of fire occurrence was made in Durango, one of the most affected states in the country. The spatial analysis was performed using a Moran index while the temporal analysis was done through the analysis of stationary and autocorrelation coefficients. A Geographically Weighted Regression was used to determine the most important factors that affect fire size. Results indicate that fires follow an aggregated distribution and are no-stationary temporally. Fire size is strongly influenced by road density and access, which confirms the importance of the anthropogenic factors. Low precipitation and high temperatures are also climatic drivers of fire size. Overall, considering the anthropogenic factor, more support is necessary to increase education and public awareness of fire effects.
\end{abstract}

\section{KEYWORDS:}

Autocorrelation, stationarity, Geographically Weighted Regression, property regimes, temperate forests.

1 Instituto Politécnico Nacional. CIIDIR Durango. Sigma 119, Fracc. 20 de Nov. II. Durango, Dgo.

Autor para correspondencia: guperezv@ipn.mx 


\section{INTRODUCCIÓN}

Los incendios forestales en bosques de clima templado frío pueden ser por un lado uno de los agentes más destructivos, pero por otro pueden ser uno de los factores más positivos que contribuyen al establecimiento y desarrollo de la vegetación forestal (Oliver y Larson, 1996; Rodríguez-Trejo y Fulé, 2003). Cuando los regímenes son alterados, los incendios forestales contribuyen a la emisión de gases efecto invernadero, estimulan la aparición de especies invasivas, modifican el flujo de agua y son de gran riesgo para la biodiversidad y la vida humana (Shlisky et al., 2007). Combinados con otros agentes climáticos, como sequías prolongadas y fuertes vientos, los efectos de los incendios son tan devastadores que la recuperación de las áreas toma grandes periodos de tiempo. La exclusión del fuego, o la ausencia total del uso del fuego en áreas forestales por periodos prolongados, también es un factor que contribuye a la generación de incendios forestales de gran magnitud debido a la acumulación de material combustible (Oliver y Larson, 1996). En su aspecto positivo, los incendios forestales son de gran importancia para muchas coníferas y latifoliadas, ya que influyen en la regeneración, reproducción, competencia, nutrición, aclareo, saneamiento y sucesión de ciertas especies forestales (Oliver y Larson, 1996). Algunas especies intolerantes de pinos requieren por ejemplo de la presencia del fuego para expeler la semilla que se encuentra en sus órganos reproductivos, abrir espacios del estrato superior o limpiar el mantillo orgánico del suelo para favorecer la germinación (Rodríguez-Trejo y Fulé, 2003).

En México, la mayoría de los incendios forestales se presentan en la primavera y el periodo de ocurrencia se extiende hasta que la vegetación, estimulada por las primeras lluvias, reinicia su periodo de crecimiento y desarrollo (Rodríguez-Trejo y
Fulé, 2003; Alanís-Rodríguez et al., 2008). La ocurrencia de los incendios depende de muchas variables climatológicas y topográficas (precipitación, altitud, humedad, temperatura y exposición, entre otras) que dependen a su vez de la variabilidad temporal (Drury y Veblen, 2008) y por lo general no tienen un patrón de distribución espacial aleatorio (Ávila et al., 2010a). Normalmente, años húmedos dan origen a la formación de una densa y rica cobertura vegetal que eventualmente es el combustible ideal para la ocurrencia de incendios terrestres de mediana a relativamente alta intensidad en el año seco siguiente (Fulé y Covington, 1999). De la misma manera, las áreas con orientación norte y de alta elevación tienen menor riesgo de presencia de incendios, mientras que aquellas con orientación sur y suroeste generalmente son más susceptibles a la presencia de incendios (Fulé y Covington, 1999).

Las actividades humanas son un factor muy importante que ha influido en la ocurrencia de los incendios forestales en muchas partes de México (Ávila et al., 2010b). Muchos agricultores utilizan el fuego como la principal herramienta para limpiar los terrenos que son utilizados a su vez para el cultivo de productos básicos como el maíz y frijol o para propiciar el rebrote de pastizales. El mal uso del fuego frecuentemente genera la dispersión de grandes y severos incendios que no sólo destruyen la flora y fauna silvestre, sino que además afectan directamente a la población en general (Rodríguez-Trejo y Fulé, 2003). Cuando el fuego es usado recurrentemente en las mismas áreas se genera una baja acumulación de material combustible y da origen a incendios de baja intensidad. En contraste, cuando se presentan en áreas grandes y dispersas y de manera esporádica, hay grandes posibilidades de que se generen incendios de moderada a alta intensidad capaces de eliminar completamente la cobertura arbórea (Fulé y Covington, 1999). 
El objetivo general de este estudio es hacer un análisis espacio-temporal de los incendios presentados en el estado de Durango en el periodo 2000-2011. El enfoque en Durango se debe a los grandes impactos que tienen los incendios en esta entidad. De acuerdo con las estadísticas de la Comisión Nacional Forestal (Conafor), en ese periodo se quemaron alrededor de 136000 ha, lo que lo posiciona como uno de los cuatro estados con mayor afectación en el país. De la misma manera, a nivel nacional la superficie promedio por incendio fue de 31 ha por incendio, mientras que en Durango la superficie promedio fue de 72 ha por incendio, con algunos extremos hasta de 300 ha por evento. Estas cifras sugieren que los incendios en Durango, a diferencia de otras entidades, se presentan en menor número pero son más difíciles de controlar. Algunos factores que pueden explicar estas diferencias son la difícil accesibilidad, topografía, clima $\mathrm{y}$, desde luego, la interacción del hombre con el bosque. De manera particular, el estudio trató de evaluar los patrones de distribución de los incendios en este periodo y la influencia de la estacionalidad temporal, así como identificar las variables más importantes que influyen en el tamaño de los incendios. Se hipotetiza que la magnitud de los incendios está fuertemente influenciada por la precipitación, temperatura y otros factores de carácter antropogénico como la cercanía a caminos y poblados.

\section{METODOLOGÍA}

Para llevar a cabo estudios que simultáneamente integren el tiempo y espacio de fenómenos como los incendios forestales, se requiere el conocimiento de estadísticas no convencionales y la información de series de tiempo. Por un lado, las técnicas estadísticas convencionales, con las cuales se asume que las observaciones son independientes, generalmente no aplican al análisis espacial debido a la gran similitud que existe entre puntos o polígonos vecinos (Burt y Barber, 1996). La similitud entre vecinos y su alto grado de dependencia ha sido sustentado claramente en la primera ley de la geografía que menciona que todos los puntos están relacionados entre sí, pero aquellos más cercanos tienen mayor relación (Tobler, 1970). En este caso se requiere la aplicación de herramientas estadísticas donde este supuesto no sea enteramente violado y se pondere la cercanía (o lejanía) de las observaciones (Hines y Hines, 1979; Brady e Irvin, 2011). Ejemplos de este tipo de herramientas son la regresión geográficamente ponderada (Fotheringham et al., 2002;), la cual se explica en detalle más adelante. Por otro lado, la información de series de tiempo para el análisis temporal, muchas veces restringida por la falta de datos confiables, también requiere la aplicación de métodos estadísticos complejos (como los modelos autoregresivos o de promedio variante) en donde el valor observado en un punto del tiempo trata de predecir el comportamiento de otro valor en otro punto del tiempo (Burt y Barber, 1996).

\section{Área de estudio}

El estudio se desarrolló en los bosques de clima templado-frío del estado de Durango, esto es en el macizo montañoso conocido como Sierra Madre Occidental, cubierto en su mayoría por bosques de coníferas y latifoliadas. Durango es el estado del país con mayores reservas forestales de asociaciones de pinos $y$ pinos-encinos (Fig. 1) y uno de los más afectados por los incendios forestales (Conafor, 2012). Cuenta con una gran diversidad topográfica, lo que lo hace importante para estudiar la variabilidad espacial de la ocurrencia de los incendios, y su extensión territorial permite ver el papel de los propietarios de los terrenos 


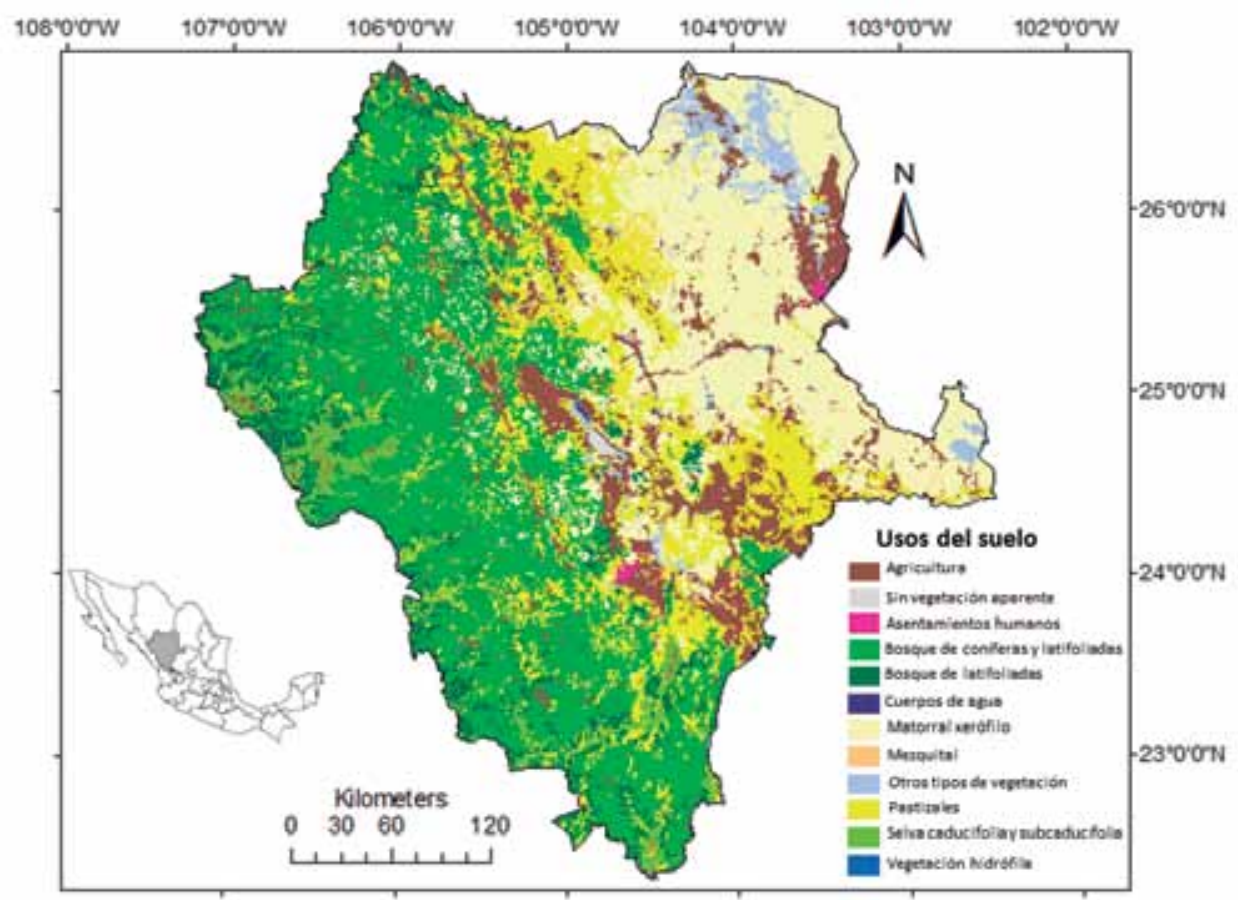

Figura 1. Localización del estado de Durango y los principales tipos de uso del suelo, incluyendo los bosques de coníferas y latifoliadas. Estos bosques constituyen el área de estudio de este trabajo (Fuente: con información del INEGI).

forestales en la prevención, control y combate de incendios forestales. Aproximadamente $80 \%$ de la superficie forestal es propiedad ejidal y su manejo depende en gran medida de la organización de estas comunidades. El área de estudio revela la existencia de cuatro zonas climáticas marcadas: a) climas secos y semisecos en las estribaciones al oriente, norte y noroeste del área; $b$ ) climas templados y semifríos en la parte alta y parte media, semisecos hacia la vertiente oriental y subhúmedos hacia la occidental; c) semicálidos en la vertiente occidental; y $d$ ) cálidos en las partes bajas y cañadas del área (González-Elizondo, 2012). La topografía es muy accidentada y da origen a varias corrientes hidrológicas. Una de ellas es la quebrada del río Mezquital, la cual atraviesa la parte montañosa entre el norte de
Nayarit y el sur de Durango, y aunque nace en la parte oriental de la zona montañosa, desemboca en el Pacífico. Los picos más altos son Cerro Gordo (3347 m), Barajas (3310 m), El Huehuento (3262 m) y el cerro de Las Antenas (3224 m) (González-Elizondo, 2012).

\section{Análisis espacial}

Se hizo un análisis de la distribución espacial de los incendios forestales en Durango con una medida de la autocorrelación basado en el índice de Moran (Moran, 1950). La autocorrelación espacial tiene sus bases en la primera Ley de la Geografía que menciona que todos los puntos están relacionados entre sí, pero aquellos más cercanos tienen mayor relación 
(Tobler, 1970; Hines y Hines, 1979). Esta medida evalúa la correlación de una variable con ella misma a través del espacio (Wong y Lee, 2005); si la distribución espacial de esa variable tiene algún patrón distintivo (no aleatorio), se dice que está espacialmente autocorrelacionada. El índice de Moran $I_{M}$ es uno de los índices más comunes en la medición de la autocorrelación espacial y compara el valor de una variable $x$ en un determinado punto $i$ con los valores de la misma variable en otro punto geográfico $j$,

$$
I_{M}=\frac{n \sum_{i} \sum_{j} w_{i j}\left(x_{i}-\bar{x}\right)\left(x_{j}-\bar{x}\right)}{\sum_{i} \sum_{j} w_{i j} \sum_{i}\left(x_{i}-\bar{x}\right)^{2}}
$$

donde:

$n$ es el numero de datos, $\bar{x}$ es el valor promedio y $w_{i j}$ toma el valor de uno si los puntos $i$ y $j$ son adyacentes o cero si son no adyacentes. El $I_{M}$ puede tomar valores de -1 a 1 , donde los valores negativos significan que la variable tiene un patrón de distribución disperso y los positivos tienen un patrón de distribución agregado. Si el índice se aproxima a cero, entonces la variable se dice que tiene un patrón de distribución aleatorio (Wong y Lee, 2005). Para probar la hipótesis nula de no autocorrelación, la distribución empírica se compara contra una distribución teórica, utilizando una prueba de significancia (Burt y Barber, 1996):

$$
\mathrm{Z}\left(I_{M}\right)=\frac{I_{M}-E\left(I_{M}\right)}{S_{E\left(I_{M}\right)}}
$$

donde:

$E I_{m}$ es el valor esperado de $I_{m}$ y $S E_{(I m)}$ es la desviación estándar. La hipótesis nula no se puede rechazar si el estadístico $Z\left(I_{M}\right)$ es menor que la distribución teórica a un determinado nivel de confiabilidad (por lo general, igual a 0,05) (Burt y Barber, 1996).

El coeficiente C de Geary (Geary, 1954) es también otra medida común- mente usada para estimar la autocorrelación espacial. Al igual que el índice de Moran, el coeficiente C de Geary utiliza pares de datos para comparar la relación de un valor observado con sus vecinos (Fortin et al., 1989). Sin embargo, mientras que el índice de Moran utiliza el promedio de las desviaciones de los pares de datos, el coeficiente $G$ de Geary usa el valor directo de esas desviaciones (Wong y Lee, 2005). En este estudio se empleó el índice de Moran dado que su distribución numérica es más cercana a los datos observados que el coeficiente $C$ de Geary (Cliff y Ord, 1981; Fortin et al., 1989; Overmars et al., 2003).

Para verificar la variabilidad del índice de Moran a diferentes distancias, se utilizó un correlograma que relaciona tanto la distancia como el valor de autocorrelación. El correlograma provee evidencia de la intensidad de autocorrelación, el tamaño de la zona de influencia y el tipo de distribución espacial de una variable bajo estudio (distribución de incendios) (Fortin et al., 1989). A diferencia de los semivariogramas, que también sirven para analizar la autocorrelación espacial a diferentes distancias, los valores de un correlograma (p. ej. índice de Moran) pueden ser probados estadísticamente $y$, dado que utiliza valores estandarizados, puede analizar varios casos simultáneamente (Overmars et al., 2003).

\section{Análisis temporal}

El análisis temporal consistió básicamente en probar dos propiedades de los fenómenos estocásticos basados en series de tiempo: estacionalidad y autocorrelación temporal. Dado el número de años incluidos en el estudio, no se construyeron modelos de simulación usando procesos autoregresivos o de promedio variante. La estacionalidad temporal se define como aquellos procesos en los cuales sus 
momentos estadísticos (media, varianza, etc.) no son constantes a través del tiempo (Burt y Barber, 1996). Para probar esta propiedad se construyeron funciones de densidad de probabilidad de la variable de interés $Y_{t}$ (esto es, superficie afectada por incendio) para cada año $t$. El efecto de estacionalidad temporal se presenta si las funciones de probabilidad son las mismas para todos los años. Esto es, si la varianza $\sigma_{t}^{2}$ y media $\mu_{t}$ son constantes para todos los años. En este caso,

$$
\sigma_{t}^{2}=E\left(Y_{t}-\mu_{t}\right)^{2}
$$

y

$$
\mu_{t}=E\left(Y_{t}\right)
$$

Ejemplos de las funciones de probabilidad usadas fueron: Weibull, Pearson, Normal, Log-Normal, entre otras.

De la misma manera que la autocorrelación espacial, también se analizó si existe dependencia entre observaciones cercanas en el tiempo. La autocorrelación temporal mide el grado de dependencia de una variable de interés $Y$ en un tiempo $t$ con ella misma en un tiempo $t+k$ (Burt y Barber, 1996); donde $k$ representa el número de periodos de evaluación (en inglés, denominados como lags) y puede tomar diferentes valores. La autocorrelación para el periodo $k=1$, por ejemplo, significa correlacionar la variable $Y_{t}$ con la misma variable $Y_{t+1}$ en cuyos datos han sido desplazados una unidad de tiempo. La autocorrelación temporal se expresa entonces como:

$$
\rho(t, t+k)=\frac{E\left(Y_{t}-\mu_{t}\right)\left(Y_{t+k}-\mu_{t+k}\right)}{\sigma_{t} \sigma_{t+k}}
$$

donde:

$\sigma$ es la desviación estándar (los otros términos han sido definidos anteriormente). En este caso, $\rho$ también toma valores de $1 \mathrm{a}-1$, donde el valor positivo sugiere una alta correlación y el valor negativo indica que la variable $Y$ tiende a oscilar con valores arriba del promedio, seguidos inmediatamente por valores abajo del promedio. Cuando tiende a cero, significa que no hay correlación en el periodo de tiempo evaluado $k$ (Burt y Barber, 1996). En el caso de los incendios forestales, la autocorrelación temporal significa que la magnitud de un incendio (esto es superficie afectada o número de eventos por unidad de área) depende en gran medida de la magnitud que se haya presentado el día, mes o año anterior. Si no hay autocorrelación, significa entonces que su magnitud se manifiesta de forma aleatoria.

\section{Factores que afectan la magnitud de los incendios}

Una vez que se detectó el patrón de distribución de los incendios y se evaluó si hay o no autocorrelación espacial y temporal, se procedió a identificar los factores más importantes que influyen en la magnitud de los incendios forestales. Se utilizó el modelo de regresión geográficamente ponderado (RGP) que asume de igual manera que todos los puntos están relacionados entre sí, pero aquellos más cercanos tienen mayor relación, esto es la primera Ley de la Geografía. A diferencia de la regresión lineal simple, el modelo RGP asume que cada variable independiente afecta la variable dependiente en diferentes proporciones o pesos geográficos (lo cual se conoce como efecto no estacionario) (Fotheringham et al., 2002; Brady e Irwin, 2011). Por ejemplo, el modelo de regresión lineal asume que la altitud tiene el mismo efecto sobre los incendios forestales en el punto $u_{1}$ y en el punto $u_{2}$ donde ambos tienen la misma altitud. Sin embargo, esta forma de estimación ignora que la magnitud de los incendios puede estar influenciada por la topografía del terreno o el tipo de vegeta- 
ción que produce condiciones climáticas diferentes al mismo nivel de altitud. El resultado generaría un coeficiente de altitud con un elevado error estándar y potencialmente errores en la estimación de la superficie afectada. Al modelo de regresión lineal obtenido a través de mínimos cuadrados también se le conoce como modelo global, mientras que al método de RPG se le conoce como modelo local (Fotheringham et al., 2002).

En este estudio se usó el modelo RGP para estimar la magnitud de los incendios usando el logaritmo natural de la superficie afectada ${ }^{1}(\operatorname{lnSup})$ en función de algunas variables ambientales y topográficas. El modelo RGP tiene la expresión siguiente (Fotheringham et al., 2002):

$$
\begin{aligned}
& \operatorname{lnSup}(u, v)_{i}=\beta_{0}(u, v)_{i} \\
& +\sum_{i=1} \beta_{i}(u, v)_{i} X+\varepsilon(u, v)_{i}
\end{aligned}
$$

donde el término $(u, v)$ indica que los parámetros $\beta_{0}$ y $\beta_{i}$ (coeficiente de intercepción y pendiente, respectivamente) deben ajustarse en función de la ubicación del punto (incendio) $i$ que cuenta con las coordenadas $u$ y $v$. Esto significa que cada punto (incendio) tendrá sus propios coeficientes $\beta_{0}$ y $\beta_{i}$. La variable $X$ indica el conjunto de factores que tienen relación con la magnitud del incendio. El componente $\varepsilon(u, v)$ representa el error asociado al punto con las coordenadas $u$ y $v$. Este modelo (conocido en inglés como Geographically Weighted Regression) asume que los

1 Los incendios forestales están representados por puntos, no polígonos (según información oficial de la Conafor). En cada punto se cuenta con información sobre la superficie afectada y el tiempo que se requirió para su extinción, entre otra información. En este caso, la magnitud esta dada por la superficie afectada y se asume que el punto con dicha información es el centro del polígono. puntos son no estacionarios y que, en este caso, la magnitud de los incendios varía en función de su ubicación.

En virtud de que el RGP considera la cercanía de los puntos colindantes, es necesario definir un círculo de búsqueda de los vecinos colindantes. Este círculo de búsqueda (o ancho de banda, como se denomina comúnmente) depende de la distancia entre los puntos observados, la similitud del área (patrones de distribución) y del error que se produce durante la simulación. Uno de los métodos más comunes para definir el ancho de búsqueda es a través del Criterio de Información de Akaike (CIA) en el cual se busca minimizar su valor a través de procesos iterativos. Una vez que el ancho de la banda (o círculo de búsqueda) ha sido seleccionado, lo que sigue es el ajuste del modelo que eventualmente produce los pesos específicos para cada variable de interés. En este caso, puntos cercanos al dato observado reciben un peso mayor que aquellos más distantes. El ajuste del modelo, que involucra la identificación de los factores que afectan la magnitud de los incendios, se hizo con el apoyo de los parámetros comunes como el coeficiente de determinación, error estándar y el coeficiente CIA. Se utilizó un modelo tipo Gaussian, donde el ancho de banda fue automáticamente identificado por iteración adaptativa (ver detalles del modelo RGP en Fotheringham et al., 2002; Harris et al., 2011).

Para probar la hipótesis nula de no estacionalidad de las variables independientes se utilizó una prueba de Monte Carlo que compara la varianza observada de los parámetros estimados de cada una de las variables contra un conjunto de datos tomados aleatoriamente. Se estimaron valores de probabilidad para cada una de las variables y se usó un nivel de significancia de 0,05. El procesamiento de los datos se hizo con el programa GWR3.0 $®$ 
de los autores, Martin Charlton, A. Stewart Fotheringham y Chris Brunsdon (Fotheringham et al., 2002).

\section{Datos}

La información de los incendios fue obtenida de la base de datos de la Comisión Nacional Forestal (Conafor) y comprendió el periodo 2000-2011. Esta dependencia, junto con los gobiernos de los estados y los dueños de terrenos forestales, coordina las operaciones de prevención, control y combate de los incendios forestales en el país, y lleva un registro de eventos en un sistema de información geográfica. La base de datos incluye, además de la ubicación de los incendios, las variables dependientes: superficie afectada (logaritmo natural) y número de días requeridos para la extinción del evento. Las variables independientes fueron tomadas de otras fuentes de información. Se utilizó el modelo digital de elevación del Instituto Nacional de Geografía (INEGI) para obtener los datos de altitud y exposición. También se utilizaron mapas temáticos del INEGI para obtener información sobre vegetación, suelos, caminos y poblados. Las variables climáticas precipitación anual y temperatura media mensual fueron obtenidas según el año de ocurrencia del incendio de la base de datos del Servicio Meteorológico Nacional. En este caso, los datos se obtuvieron de la estación climatológica más cercana al incendio. Se usaron modelos de regresión lineal simple para aquellos casos en que las estaciones climatológicas estaban muy distantes de los puntos de los incendios. La variable que identifica tipos de propiedad fue obtenida del Registro Agrario Nacional.

Con la información recabada fue posible hacer transformaciones o análisis cartográficos a las variables. Por ejemplo, se calculó un índice de gravedad pobla- cional (IGP) (Poudyal et al. 2011), expresado como:

$$
I G P=\sum_{n}^{N} \frac{P_{n}}{D_{i n}^{2}}, \forall_{n}: D \leq 20 \mathrm{~km}
$$

donde:

$P$ es la población total de centro de población $n, N$ el número total de pueblos, $D$ es la distancia (radio) entre el incendio $i$ y el poblado $n$, tomados en un máximo de $20 \mathrm{~km}$ a la redonda del incendio. El IGP es una medida del efecto de la densidad de población en la ocurrencia de los incendios. Índices altos significan una alta presión humana en la ocurrencia. De igual forma, utilizando herramientas de los sistemas de información geográfica, se calcularon los promedios geográficos y desviaciones estándar de los incendios forestales en cada año, los puntos calientes (hotspots) y fríos (coldspots) determinados por el estadístico $\mathrm{G}$ General (Gettis y Ord, 1992), así como la distancia más cercana de los incendios a los centros de población y caminos. La mayoría de los análisis cartográficos se hicieron con el paquete de computo ArcGis $®$. En la tabla 1 se detallan las características de las variables utilizadas en el estudio.

\section{RESULTADOS Y DISCUSIÓN}

En el periodo que se analizó en este estudio (2000-2011), se registraron 1,564 incendios que afectaron 136,370 hectáreas, lo que da en promedio 87,2 ha/ incendio. De la superficie total afectada, aproximadamente $44 \%$ corresponde a zonas de pastizales, $39 \%$ a vegetación arbustiva y el resto se distribuye en áreas con arbolado maduro y renuevo. Para detectar, combatir y extinguir los siniestros presentados, se requirieron en promedio 18,7 días. 


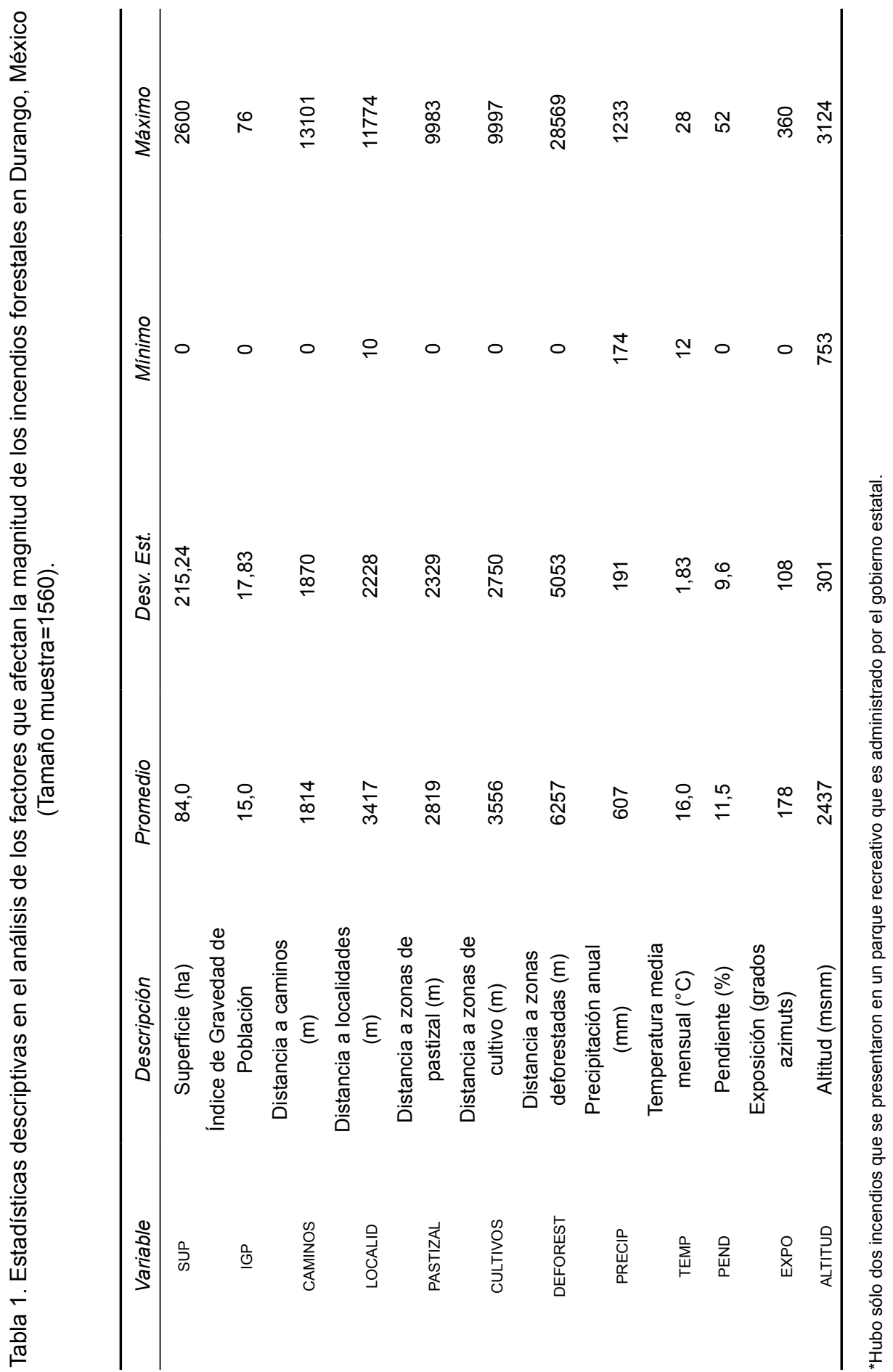




\section{Análisis espacial}

Se utilizó el índice de Moran $\left(I_{M}\right)$ para estimar un coeficiente de autocorrelación espacial que se mide por la cercanía de los incendios entre sí. Este índice se estimó para las coordenadas X y Y (longitud y latitud) y en ambos casos el coeficiente resultó con un valor positivo en promedio de 0,88 y 0,74 , respectivamente. La prueba de hipótesis nula de no autocorrelación fue rechazada debido a que el coeficiente $Z\left(I_{M}\right)$ fue de 6,14 para $X$ y 5,22 para $Y$. Esto significa que los incendios tienen un patrón de distribución agregado y, como se muestra en la figura 2 , con alta concentración en los caminos existentes. Se construyó un correlograma que relaciona el índice de Moran a diferentes dis- tancias, tanto para latitud como para longitud. La figura 3 muestra la forma del correlograma y sugiere que tanto a distancias cortas como lejanas, la distribución de los incendios mantiene un patrón de distribución de forma agregada. Ávila et al., (2010b), en su estudio de incendios forestales en Durango, encontraron que la distribución de los incendios tampoco sigue un patrón aleatorio. Aunque ellos utilizaron una base de datos más pequeña, sus resultados coinciden con los presentados en este trabajo.

En el análisis de los promedios geográficos anuales, o centros de concentración, se puede observar también que, con excepción de 2011 que fue un año con muy baja precipitación, el centro geográfico

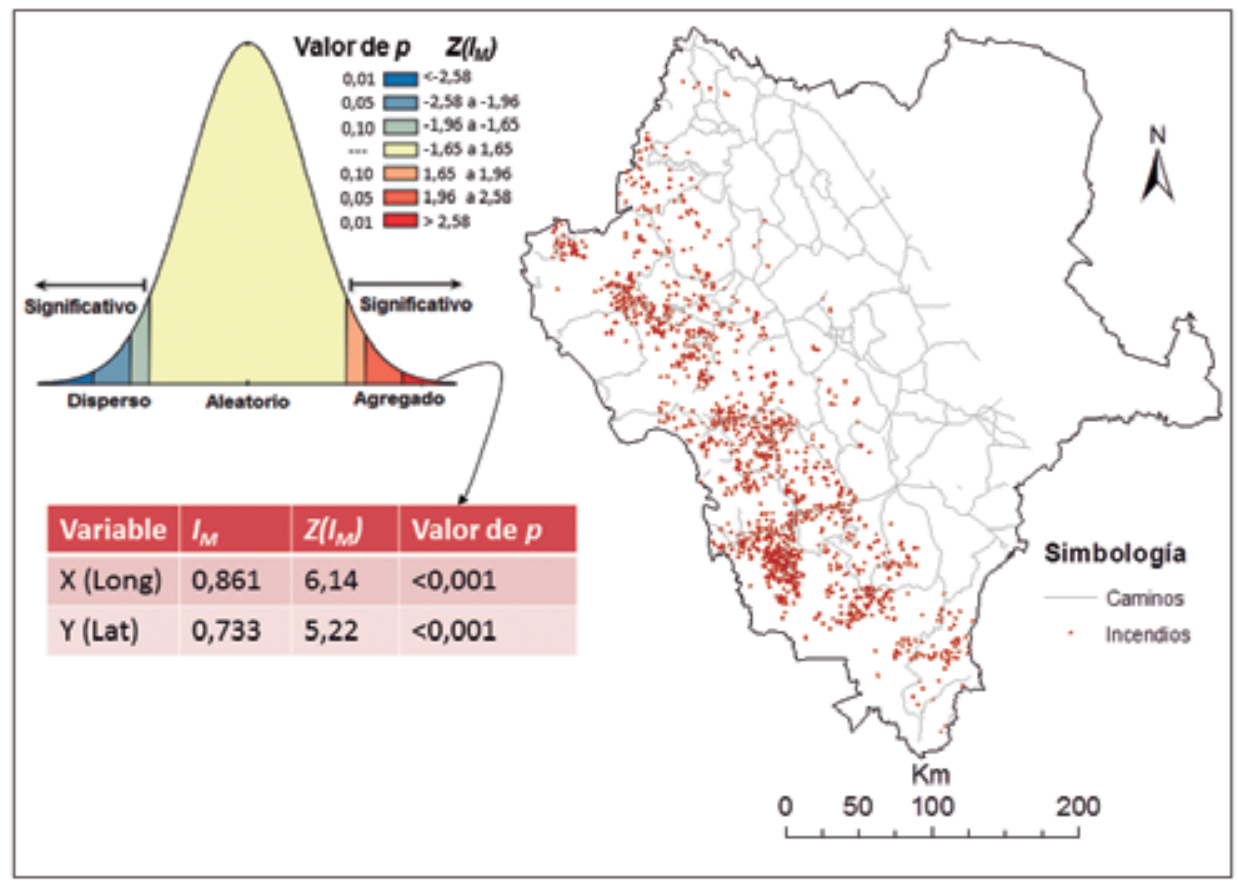

Figura 2. Índice de Moran para las variables $X$ (long) y $Y($ Lat) y patrón de distribución de los incendios registrados en el estado de Durango. La figura indica que los incendios se distribuyen de manera agregada. 


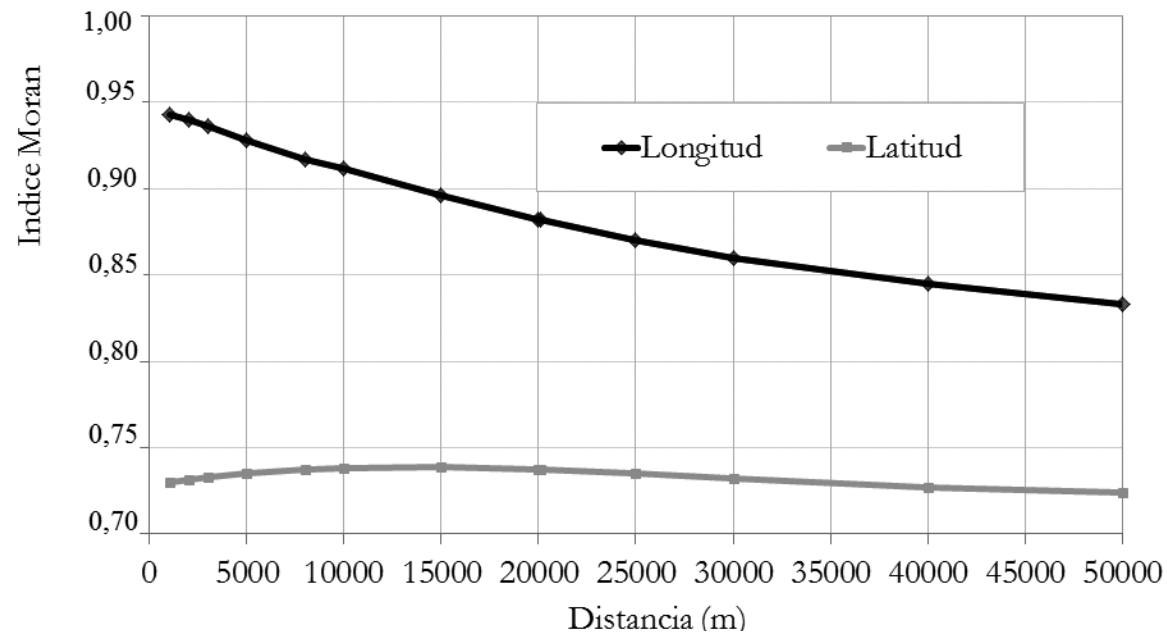

Figura 3. Correlograma de los índices de Moran para latitud y longitud de los incendios forestales registrados en el periodo 2000-2011 en el estado de Durango, México. Todos los valores del índice de Moran fueron estadísticamente significativos $(p<0,01)$.

ha estado desplazándose ligeramente hacia el centro-sur del estado, esto es en dirección a la ciudad de Durango. La dispersión se da a lo largo del eje de la Sierra Madre Occidental, en dirección Norte-Sur, que es la parte donde mayoritariamente se distribuyen los recursos forestales (Fig. 4). Algunas de las posibles explicaciones de este movimiento pueden ser que existe una mayor concentración de la población (cerca de $40 \%$ ) en la parte centro-sur del estado. En contraste con sólo $20 \%$ que habita en la parte norte. Como se ha comentado, el hombre juega un papel importante en la ocurrencia de los incendios. De acuerdo con el gerente estatal de la Conafor, alrededor de $80 \%$ de la superficie afectada por estos eventos ha sido por descuidos humanos (Olayo-González, 2012). Otra posible explicación de la ubicación de los centros geográficos es la infraestructura caminera que existe en la parte centro-sur, que incluye caminos pavimentados como las carreteras libre $y$ de cuota Durango-Mazatlán y la de San Miguel de Cruces, que permiten tener un mejor acceso a las áreas forestales. El proceso de colecta de información también puede ser otra causa del movimiento de los centros hacia el sur del estado. De acuerdo con el gerente estatal de la Conafor, muchos incendios son controlados por los propietarios sin hacer los reportes correspondientes. Es posible que la información, especialmente de la zona norte, no se recabe de la misma manera que la de la zona sur.

Para analizar áreas críticas de presencia de incendios se utilizó el Estadístico G General para identificar focos calientes en cuanto a la superficie afectada y al número de días requeridos para su extinción. La figura 5 muestra los 'focos rojos' (hotspots) o lugares con alto impacto por los incendios forestales, así como aquellos 'focos fríos' donde el efecto es menor. Existen entre 5 y 6 focos rojos en la entidad y se localizan en lugares poco accesibles y lejanos a los centros de población. Estos son: la zona oeste del municipio de Tepehuanes y suroeste de Guanaceví, la parte central del municipio de Santiago Papasquiaro, Otáez, parte 


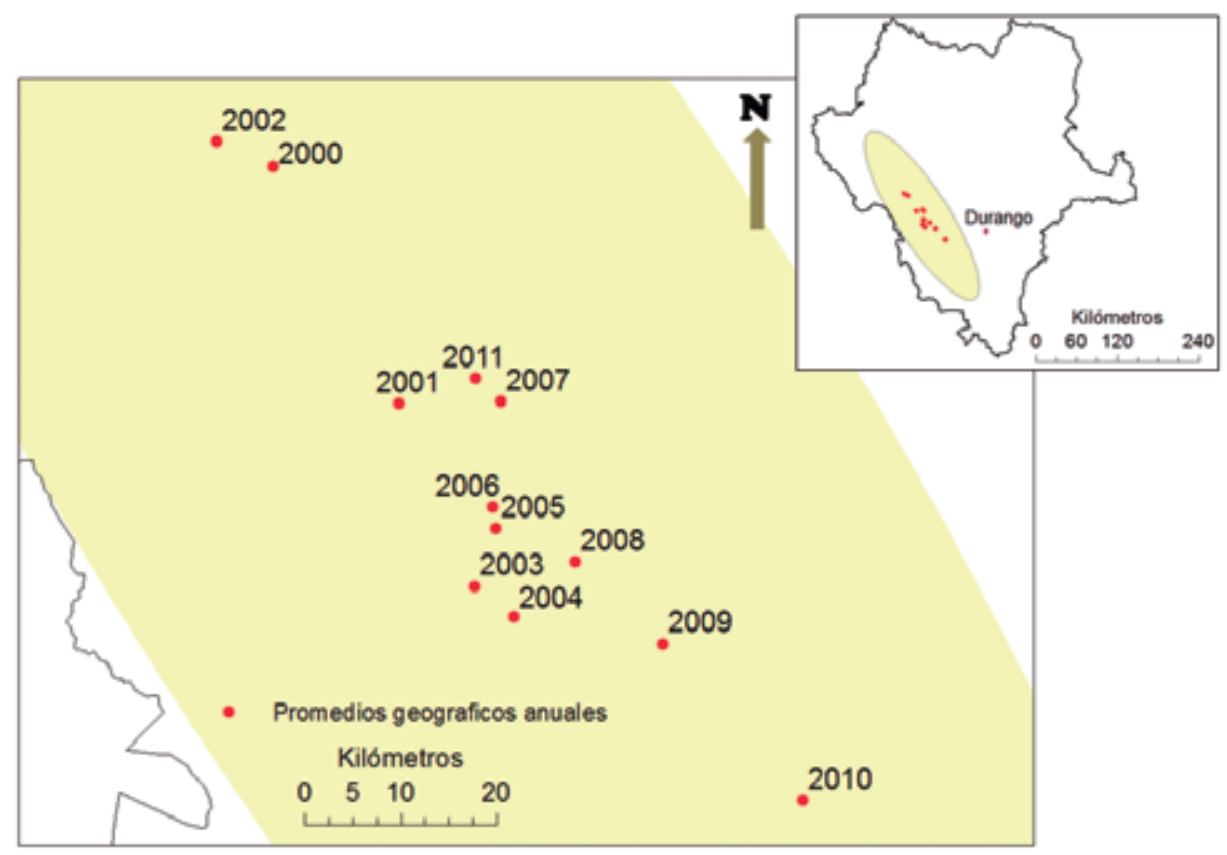

Figura 4. Promedios geográficos anuales de los incendios registrados en Durango para el periodo 2000-2011. La figura elíptica muestra la orientación y desviación estándar de todos los incendios.

norte-centro del municipio de Durango y el paraje conocido como La Flor, norte de Mezquital. En términos del número de días que se requieren para la extinción, hay un área crítica más que se localiza en la parte sur de los municipios de Pueblo Nuevo y Mezquital. En contraste, los 'focos fríos' se localizan alrededor de los núcleos poblacionales de El Salto, Pueblo Nuevo y San Miguel de Cruces, San Dimas.

Es importante mencionar que los focos fríos se ubican en las áreas donde actualmente existe una alta participación de los propietarios de los terrenos forestales. La zona de San Miguel de Cruces, municipio de San Dimas y El Salto, municipio de Pueblo Nuevo, se caracterizan por tener una alta organización social, donde la participación de los dueños y poseedores, no sólo en el combate de incendios, sino en todas las actividades de restauración y manejo, es muy positiva. Si bien es cierto que existe una alta frecuencia de incendios, éstos son controlados más eficientemente, es decir, en menor tiempo y con una menor superficie afectada.

\section{Análisis temporal}

Para evaluar la estacionalidad temporal se construyeron funciones de densidad de probabilidad de la variable de interés (esto es, superficie afectada por incendio) para cada año. Se estimó el promedio y la varianza y se analizó la homogeneidad de varianzas mediante la prueba de Levene. 


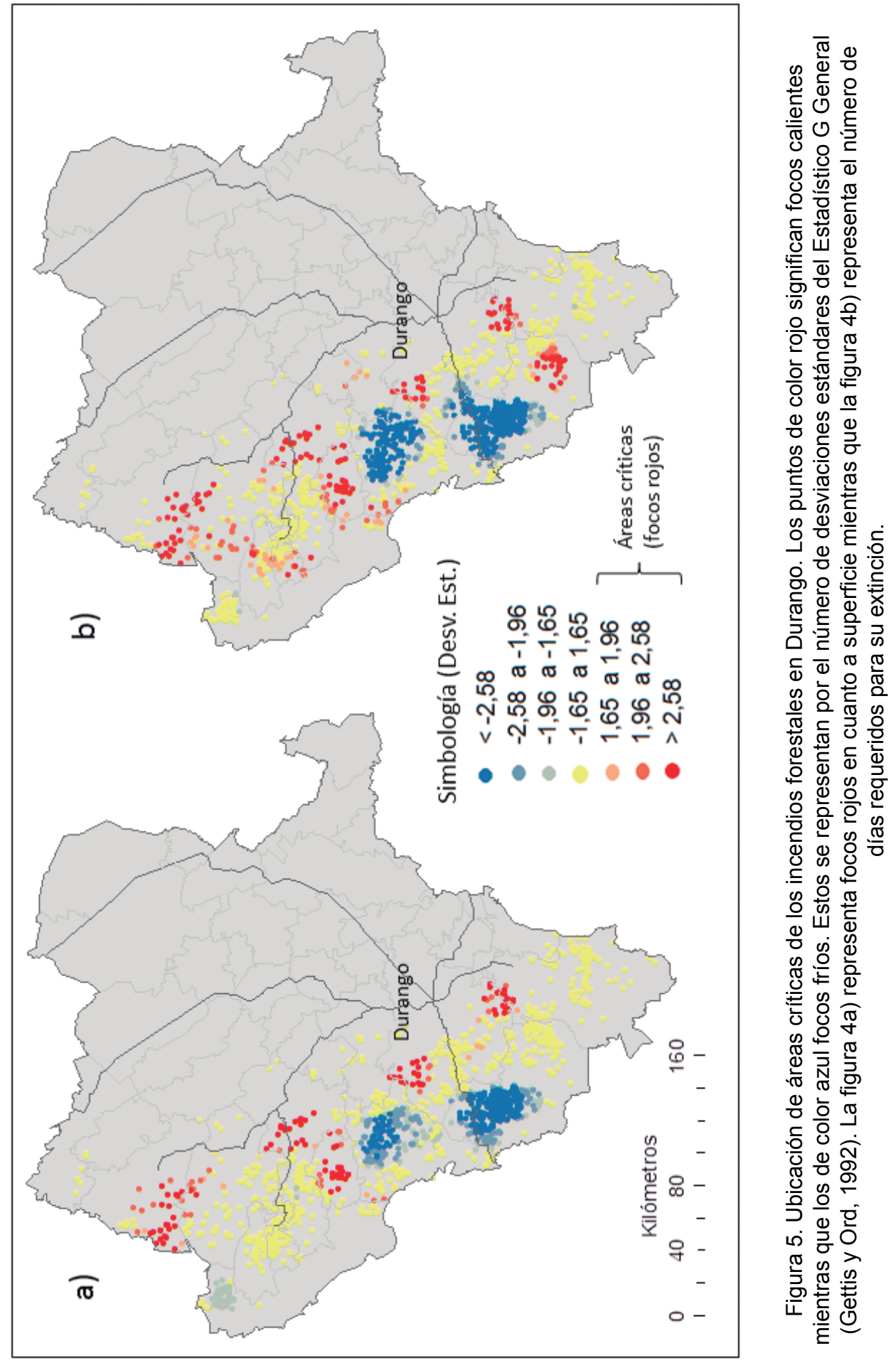


Los resultados indican que la función de densidad de probabilidad mas común fue la de Log-Pearson III, la cual se repite en 5 de los 12 años que comprende el estudio. Sin embargo, el promedio y la varianza en cada uno de los años es diferente (Tabla 2).

La prueba de Levene y el análisis de varianza de una vía muestran que tanto la varianza $(L=18,012 ; p<0,001)$ como el promedio de la superficie afectada $(F=8,995 ; p<0,001)$, respectivamente, son estadísticamente diferentes. La excepción se observa en el periodo 2004-2005, en donde se obtuvo la misma densidad de probabilidad (Log-Pearson III).

Sin embargo, la prueba de t revela que sí hay diferencias significativas en las varianzas y los promedios de estos dos años. Por lo tanto, los resultados muestran que en el periodo evaluado no existe homogeneidad de varianzas en la información de superficie afectada por los incendios forestales y que este fenómeno es un proceso no estacionario temporalmente. En otras palabras, el tamaño de

Tabla 2. Funciones de distribución de probabilidad para la superficie afectada (ha) por los incendios registrados en el periodo 2000-2011 en Durango.

\begin{tabular}{|c|c|c|c|c|c|c|}
\hline \multirow[b]{2}{*}{ Año } & \multirow[b]{2}{*}{ \# Eventos } & \multirow[b]{2}{*}{ Rango } & \multirow[b]{2}{*}{ Promedio } & \multirow[b]{2}{*}{ Varianza } & \multicolumn{2}{|c|}{ Función de distribución } \\
\hline & & & & & Estadístico* & Tipo \\
\hline 2000 & 132 & 4995 & 213,8 & 265360 & 0,247 & Pearson 6 \\
\hline 2001 & 66 & 799 & 87,2 & 20822 & 0,699 & Weibull \\
\hline 2002 & 170 & 799 & 70,4 & 10852 & 0,291 & Log-Pearson III \\
\hline 2003 & 162 & 679 & 52,9 & 7461 & 0,405 & Lognormal \\
\hline 2004 & 116 & 429 & 28,6 & 4010 & 0,697 & Log-Pearson III \\
\hline 2005 & 230 & 1461 & 54,5 & 17171 & 1,755 & Log-Pearson III \\
\hline 2006 & 140 & 399 & 65,8 & 8220 & 0,624 & Fatigue Life \\
\hline 2007 & 88 & 529 & 41,8 & 6424 & 0,309 & Wakeby \\
\hline 2008 & 116 & 1898 & 99,4 & 65569 & 0,423 & Log-Pearson III \\
\hline 2009 & 124 & 550 & 28,8 & 5653 & 1,345 & Wakeby \\
\hline 2010 & 43 & 1199 & 78,1 & 45555 & 0,319 & Dagum \\
\hline 2011 & 177 & 2599 & 196,0 & 200730 & 1,169 & Log-Pearson III \\
\hline
\end{tabular}

* $\quad$ En función del tipo de distribución probada. Nivel de confiabilidad igual a 95\%. 
los incendios se manifiesta de manera totalmente aleatoria en cada año. Este fenómeno es similar a la precipitación, ya que puede presentarse un año muy húmedo, pero el siguiente muy seco.

Para observar variaciones temporales a mayor detalle se estimó el coeficiente de autocorrelación temporal, el cual mide el grado de dependencia de la superficie afectada en un tiempo $t$ con ella misma en un tiempo $t+k$. Se utilizaron varias categorías y periodos $k$. Se estimó un coeficiente diario, mensual y anual y en cada categoría se utilizaron hasta 10 periodos (lags) para observar la consistencia de los resultados.

Por ejemplo, para la correlación diaria se evaluaron periodos de uno hasta diez días. En el caso de la correlación mensual se evaluaron periodos de uno hasta tres meses y en la correlación anual los periodos fueron de uno hasta cinco años.

Los resultados indican que en la correlación diaria se encontró un coeficiente que varía entre 0,08 y $0,20(p<0,05)$, lo cual indica que sí existe autocorrelación, pero ésta es muy baja. En el caso de la autocorrelación mensual y anual, a pesar de tener coeficientes relativamente altos $(0,33$ a $-0,77)$, éstas no fueron estadísticamente significativas en ninguno de los periodos $(p>0,05)$ (Fig. 6).

Estos resultados indican que hay una ligera autocorrelación diaria, esto es, la cantidad de superficie que se quema en un día, motivada quizá por las condiciones climáticas diarias, influye de forma positiva en la cantidad de superficie que se quema en el día siguiente. Sin embargo, esto no sucede con la autocorrelación mensual y anual, en las cuales, en concordancia con la prueba de Levene, la superficie afectada se manifiesta de manera aleatoria.

\section{Factores que afectan la magnitud de los incendios}

Una vez que se detectó que existe autocorrelación espacial (esto es, un patrón de distribución agregada) y se determinó que los incendios son no estacionarios temporalmente, se procedió a identificar los factores que afectan la magnitud de los incendios forestales. Primero se hizo una comparación de la regresión global (regresión lineal simple, RLS) contra la regresión local (regresión geográficamente ponderada, RGP). El modelo global arrojó un coeficiente de determinación ajustado de $30 \%$ y un valor de CIA de 5566 , mientras que el modelo local tuvo un coeficiente de determinación de $45 \%$ y un valor CIA de 5330. Los resultados indican que el modelo local tuvo una mejora de 236 puntos sobre el modelo global en términos del CIA, mientras que en términos de la varianza explicada, el modelo local (RGP) explicó $15 \%$ más en la variación de la superficie afectada. La prueba de $F$ indica también que la reducción de la suma de residuales entre el modelo global y local fue significativa $(p<0,05)$. Es evidente que el modelo local tuvo mejores resultados en su ajuste que el modelo global (Tabla 3).

De acuerdo entonces con el modelo local, los resultados de las medianas de los parámetros de los factores muestran que tanto el índice de gravedad de población (IGP), como la distancia a zonas de cultivos (CULTIVOS), la precipitación (PRECIP) y la exposición (EXPO) son negativos. El resto de los parámetros de los factores toma valores positivos (Tabla 4). Esta tabla muestra también que, con base en la prueba de Monte Carlo, en los factores: IGP, distancia a caminos (CAMINOS), distancia a zonas deforestadas (DEFOREST), PRECIP, temperatura (TEMP) y altitud (ALTITUD) se rechaza la hipótesis de estacionalidad espacial y se concluye que estos factores son significativamente no 

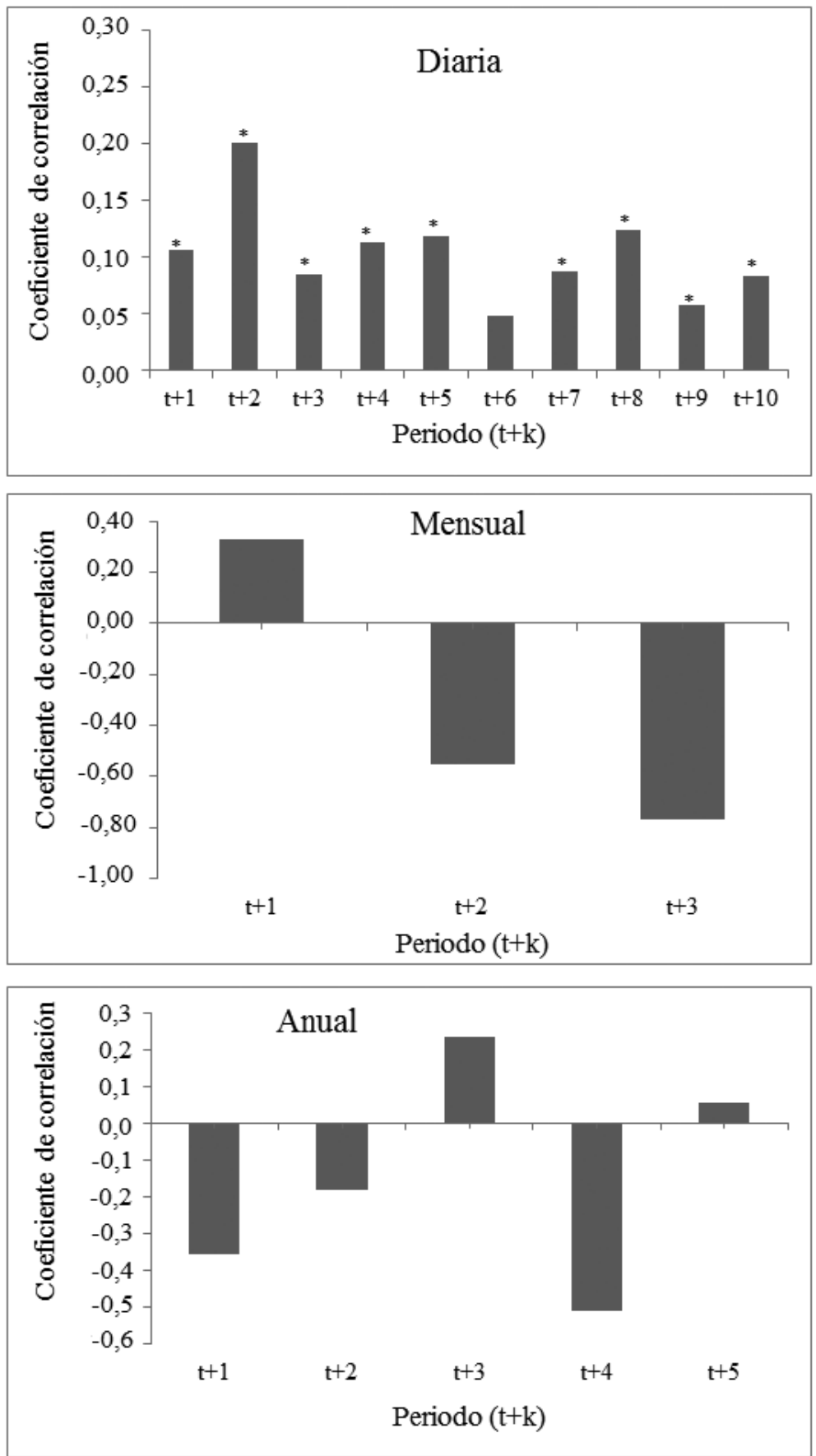

Figura 6. Coeficientes de autocorrelación temporal para la variable superficie afectada en las categorías a) Diaria, b) Mensual y c) Anual (* Significativo a 0,05). 
Tabla 3. Análisis de varianza de los modelos global (RLS) y local (RGP) para la superficie afectada por incendios forestales en Durango, México.

\begin{tabular}{ccccc}
\hline Fuente & $\begin{array}{c}\text { Suma de } \\
\text { cuadrados }\end{array}$ & $\begin{array}{c}\text { Grados de } \\
\text { libertad }\end{array}$ & $\begin{array}{c}\text { Cuadrados } \\
\text { medios }\end{array}$ & $F$ \\
\hline Modelo global (RLS) & 3169,4 & 12 & & \\
Modelo local (RGP) & 867,8 & 120 & 7,24 & \\
Residuales (RGP). & 2301,6 & 1431 & 1,61 & $4,51^{*}$ \\
\hline
\end{tabular}

* $\quad$ Prueba de F significativa a $\alpha=0,05$; RLS: Regresión Lineal Simple; RGP: Regresión Geográficamente Ponderada.

estacionarios en el área de estudio. Por ejemplo, el tamaño de los incendios varía, desde $-0,00009$ hasta 0,00034 veces la distancia a los caminos (otros factores constantes). De la misma manera, el tamaño de los incendios varía desde $-0,00301$ hasta $-0,00008$ veces el valor de la precipitación (esto también porque su efecto depende de la ubicación del incendio) y así sucesivamente para el resto de los factores.

La característica de no estacionalidad sugiere que el tamaño de los incendios forestales no sigue un patrón constante de variabilidad y que está influenciado por la posición geográfica del incendio. Por ejemplo, los incendios que geográficamente están más cerca de los caminos tienen relativamente menor superficie afectada que aquellos en donde la distancia es más grande. Esto puede deberse a que en las zonas con mayor distancia el combate y control de los incendios se dificulta y por tanto tienden a afectar más superficie que en aquellas áreas donde el acceso es relativamente fácil. De igual manera, la magnitud de los incendios es variable en dos puntos diferentes, aun cuando estos puntos tengan la misma precipitación o temperatura. Estas diferencias no podrían ser detectadas por el modelo global (RLS) en el cual se asume que la distancia a los caminos tiene un efecto constante en la superficie afectada.

Estadísticamente, los factores que se identificaron como no estacionarios más distancia a localidades (LOCALID) son también los que mayormente influyen en la magnitud de los incendios forestales (Tabla 4). Los factores LOCALID y PRECIP muestran consistencia en los signos, es decir, no registran cambios en sus valores máximos, intermedios y mínimos. La distancia a localidades tiene una relación directa, mientras que PRECIP tiene una relación inversa con el tamaño de los incendios. Esta heterogeneidad espacial hace que la posición geográfica de los incendios y su correspondiente grado de influencia de los factores antes mencionados determinen el tamaño de los incendios. Ávila et al. (2010b) detectaron que entre los factores más importantes que inciden en la ocurrencia de los incendios se encuentran la susceptibilidad de la vegetación al fuego, intensidad en el cambio de uso del suelo y la precipitación. En el caso de la precipitación, factor común en nuestro estudio, encontraron que este factor tiene una influencia directa en el número de incendios. 


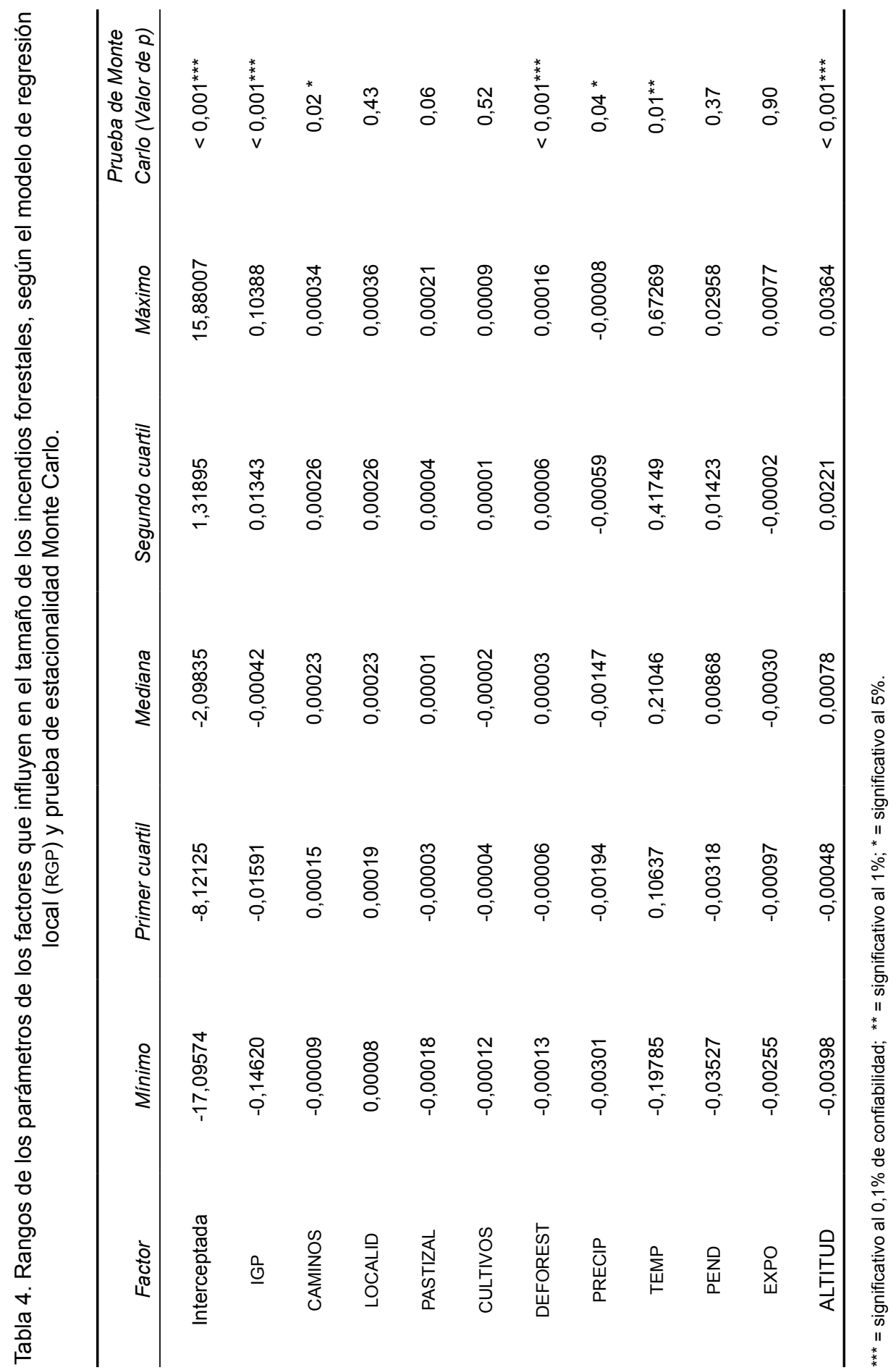


Estos resultados confirman que el tamaño de los incendios está influenciado no sólo por factores ambientales (precipitación, temperatura y altitud), sino también por factores antropogénicos (índice de gravedad de población, distancia a caminos, distancia a localidades, distancia áreas deforestadas). Y que además su efecto es variable en función de la ubicación de los incendios. Es poco lo que se puede hacer para mitigar el efecto de los primeros, pero es mucho lo que se puede hacer para reducir el efecto de los segundos. Si bien es cierto que los incendios que se localizan cerca de los caminos y poblados son de magnitud baja, su frecuencia constante y recurrente incrementa el costo de oportunidad y disminuye la posibilidad de atender otras prioridades. En el lado opuesto, los incendios que ocurren en áreas remotas o de baja densidad caminera no son muy frecuentes, pero sus efectos son tan fuertes que pueden traer consecuencias irreparables. En 1998, por ejemplo, un incendio que se propagó en la Reserva de la Biósfera de la Michilía, una área natural protegida al sur del estado que por muchos años no registró presencia de incendios (Fulé y Covington, 1999) y se caracteriza por tener una baja densidad de caminos en su parte central, ocasionó un incendio de grandes magnitudes que reemplazó por completo la cubierta forestal y ocasionó la muerte de uno de sus combatientes.

Las áreas deforestadas para la siembra de cultivos agrícolas es también otro factor que influye significativamente en el tamaño de los incendios. Pérez-Verdín et al. (2009) encontraron que uno de los factores más importantes que influyen en la deforestación es la agricultura de baja intensidad en áreas forestales. Debido a que el suelo no es propicio para cultivos agrícolas (como el maíz, principalmente), en pocos años el terreno desmontado se abandona y se localiza otro (o se expande el actual) para iniciar nuevamente el pro- ceso de clareo y limpia. Esa nueva área tiende por lo general a estar cerca de los caminos y poblados para disminuir el esfuerzo y los costos de producción (transporte, insumos, etc.). En ese proceso de limpia de terreno el fuego actúa como una de las principales herramientas de trabajo. Cuando desafortunadamente se combina con ciertos factores climáticos y no existe la capacidad de control, se convierte en un agente muy nocivo al ecosistema.

Algunas implicaciones que este estudio puede traer son un mejor entendimiento de los impactos de los factores que estimulan el tamaño de los incendios forestales, especialmente los de carácter antropogénico, y el rediseño de programas de respuesta en los focos rojos (hotspots), así como en las áreas adyacentes a los caminos principales, áreas cercanas a los centros de población y áreas deforestadas (cuyo uso ha sido para agricultura o ganadería, principalmente). Rodríguez-Trejo y Fulé (2003) discuten una serie de medidas que pueden implementarse para reducir el efecto negativo del fuego. Por ejemplo, citan una mayor capacitación a los usuarios del bosque (agricultores, ganaderos, recreacionistas, etc.), el mantenimiento de caminos para el transporte (que faciliten el transporte de las brigadas de combate), la aplicación de aclareos, quemas controladas, brechas cortafuego en perímetros adyacentes a los caminos, etc. Sus propuestas de manejo son divididas en los casos cuando los incendios son excesivos, normales (apropiados) o insuficientes.

\section{CONCLUSIONES}

Se realizó una evaluación espacio-temporal de los incendios forestales durante el periodo 2000-2011 en Durango, México, así como de los factores más importantes que los motivan. De acuerdo con los resultados, los incendios en el estado de 
Durango se distribuyen espacialmente de manera agregada y son no estacionarios temporalmente. El modelo de regresión geográficamente ponderado es una herramienta muy adecuada para el estudio de fenómenos como los incendios forestales. Este modelo, a diferencia del método de regresión lineal simple, asigna una ponderación diferente a medida que las observaciones incrementan su distancia y se hacen más disímiles. Ayuda también a identificar factores que tienen un efecto constante o variable de acuerdo con la posición geográfica. En este caso, los factores índice de gravedad de población (IGP), distancia a caminos (CAMINOS), distancia a áreas deforestadas (DEFOREST), precipitación (PRECIP), temperatura (TEMP) $y$ altitud (ALTITUD) son no estacionarios en el área de estudio y en el periodo de tiempo analizado. La no estacionalidad sugiere que el tamaño de los incendios forestales depende de su posición geográfica y de la influencia de los factores antes mencionados. Se recomienda ampliamente el uso de estas herramientas estadísticas variables estocásticas y profundizar más sobre la heterogeneidad espacial de estos factores.

Los factores más importantes que inciden en la magnitud de los incendios se pueden clasificar como de tipo ambiental (precipitación, temperatura y altitud) y antropogénico (distancia a caminos, distancia a localidades, distancia a zonas deforestadas e índice de gravedad de población). El enfoque de los técnicos forestales es, desde luego, en aquellos factores que pueden manipularse como la construcción y mantenimiento de caminos y en la capacitación a los usuarios del recurso forestal. El hombre actúa como el principal generador del fuego, pero es el mismo hombre y sus recursos el que puede hacer que su magnitud se reduzca o incremente. Los resultados sugieren que es necesario fomentar las acciones para concientizar y elevar el nivel de edu- cación de los usuarios de los terrenos forestales, así como ejecutar una serie de medidas para reducir la frecuencia/ tamaño de los incendios. Entre ellas están: una mayor capacitación a los usuarios del bosque (agricultores, ganaderos recreacionistas, etc.), el mantenimiento de caminos para el transporte de productos forestales, la aplicación de aclareos, quemas controladas, brechas cortafuego en perímetros adyacentes a los caminos, etcétera.

\section{AGRADECIMIENTOS}

Este estudio se desarrolló con financiamiento del Instituto Politécnico Nacional, proyecto SIP 20110943, Conacyt y de la Comisión de Operación y Fomento de Actividades Académicas (COFAA) del IPN. Expresamos nuestro agradecimiento a la Comisión Nacional Forestal (Conafor) Gerencia Estatal Durango y a la Coordinación General del Servicio Meteorológico Nacional, por los apoyos recibidos en la recolección de datos. Agradecemos también al doctor Marín Pompa García por los comentarios y sugerencias recibidas en este trabajo.

\section{REFERENCIAS}

Alanís-Rodríguez E., Jiménez-Pérez J., Espinoza-Vizcarra D., Jurado-Ybarra E., Aguirre-Calderón O.A., GonzálezTagle M.A. 2008. Evaluación del estrato arbóreo en un área restaurada post-incendio en el parque ecológico Chipinque, México. Revista Chapingo. Serie Ciencias Forestales y del Ambiente 14(2):113-118

Ávila-Flores, D.Y., M. Pompa-García y E. Vargas-Pérez. 2010a. Spatial analysis of fire occurrence in the Durango State. Revista Chapingo. Serie Ciencias Forestales 16(2):253-260. 
Ávila-Flores, D.Y., M. Pompa-García, S. Antonio-Nemiga, D.A. RodríguezTrejo, E. Vargas-Pérez y J. Santillán Pérez. 2010b. Driving factors for forest fire occurrence in Durango State of Mexico: A geospatial perspective. Chinese Geographical Science 20(6):491-497.

Brady, M. y E. Irwin. 2011. Accounting for spatial effects in economic models of land use: Recent developments and challenges ahead. Environ. Resource Econ. 48:487-509.

Burt, J.E. y G.M. Barber. 1996. Elementary statistics for geographers. 2a. ed. The Guilford Press. Nueva York, $640 \mathrm{p}$.

Cliff, A.D. y J.K. Ord. 1981. Spatial processes: Models and Applications. Pion: London.

Conafor (Comisión Nacional Forestal). 2012. Reporte nacional de incendios forestales. Publicación interna de trabajo. Conafor. Guadalajara, Mex. Disponible en http://www.mexicoforestal.gob.mx/files/120427\%20 reporte $\% 20$ nacional\%20incendios. pdf (Último acceso mayo 12, 2012).

Drury, S.A. y T.T. Veblen. 2008. Spatial and temporal variability in fire occurrence within the Las Bayas forestry reserve, Durango, Mexico. Plant Ecology 197:299-316.

Fortin, M.J, P. Drapeau y P. Legendre. 1989. Spatial autocorrelation and sampling design in plant ecology, Vegetatio 83: 209-222.

Fotheringham, A.S., C. Brunsdon y M. Charlton. 2002. Geographically weighted regression: The analysis of spatially varying relationships. $1 \mathrm{a}$. ed. John Wiley \& Sons, LTD. West Sussex, Inglaterra. 284 p.

Fulé, P.Z. y W.W. Covington. 1999. Fire regime changes in La Michilia Biosphere Reserve, Durango, Mexico. Conservation Biology 13(3):640-652.

Geary, R.C. 1954. The contiguity ratio and statistical mapping. The Incorporated Statistician 5: 115-145.

Gettis, A. y J.K. Ord. 1992. The analysis of spatial association by use of distance statistics. Geographical Analysis 24(3):189-207.

González-Elizondo, M.S., M. González-Elizondo, J.A. Tena-Flores, L. RuachoGonzález y L. López-Enríquez L. 2012. Vegetación de la Sierra Madre Occidental, México: Una síntesis. Acta Botánica Mexicana 100:351-403.

Harris, P., C. Brundson y A.S. Fotheringham. 2011. Links, comparisons and extensions of the geographically weighted regression model when used as a spatial predictor. Stoch. Environ. Res. Risk. Assess. 25:123-138.

Hines, W.G.S. y R.J. Hines. 1979. The Eberhardt statistic and the detection of nonrandomness of spatial point distributions. Biometrika 66:73-79.

Moran, P.A.P. 1950. Notes on continuous stochastic phenomena. Biometrika, 37:17-23.

Olayo-González, M.A. Gerente Estatal Conafor Durango. Comunicación Personal. 6 de noviembre. 2012.

Oliver, C.D. y B.C. Larson. 1996. Forest stand dynamics. McGraw Hill. Nueva York, 509 p. 
Overmars, K.P., G.H.J. de Koning y A. Veldkamp. 2003. Spatial autocorrelation in multi-scale land use models. Ecological Modelling, 164:257-270.

Pérez-Verdín, G., Y-S Kim, D. Hospodarski y A. Tecle. 2009. Factors driving deforestation in common-pool resources in northern Mexico. Journal of Environmental Management 90:331-340.

Poudyal, N.C., S.H. Cho, J.D. Strickland y D.G. Hodges. 2011. An analysis of forestland change on the northern Cumberland Plateau: Bridging the broad units and fine units datasets in a landuse model. In: J. Gan, S. Grado e I.A. Munn. Eds. Global Change and Forestry, economic and policy impacts and responses. Nova Science Publishers. Nueva York, p:63-75.

Rodríguez-Trejo, D.A. y P.Z. Fulé. 2003. Fire ecology of mexican pines and fire management proposal. International Journal of Wildlife Fire 12:23-37.
Shlisky, A., J. Waugh, P. González, M. González, M. Manta, H. Santoso, E. Alvarado, A. Ainuddi-Nuruddin, D.A. Rodríguez-Trejo, R. Swaty, D. Schmidt, M. Kaufmann, R. Myers, A. Alencar, F. Kearns, D. Johnson, J. Smith y D. Zollner. 2007. Fire, ecosystems and people: Threats and strategies for global biodiversity conservation. The Nature Conservancy Global Fire Initiative Technical Report 2007-2. IV Conferencia Internacional sobre Incendios Forestales. Sevilla, España. 13-17 de mayo de 2007.

Tobler, W.R. 1970. A computer movie simulating urban growth in the Detroit region. Economic Geography 46(2):234-240.

Wong W.S.D y J. Lee. 2005. Statistical analysis of geographic information with ArcView GIS and ArcGIS. John Wiley. Nueva York, 464 p. la ocurrencia de incendios forestales en Durango, México. Madera y Bosques 19(2):37-58. 